\title{
STELLAR POPULATION SYNTHESIS
}

\author{
Application To Galactic Bulges
}

\author{
N. ARIMOTO \\ Institut für Theoretische Astrophysik der Universität Heidelberg, Im Neuenheimer Feld 561, \\ W6900, Heidelberg 1, Germany \\ and
}

Institute of Astronomy, The University of Tokyo, Mitaka, Tokyo 181, Japan

\begin{abstract}
.
The stellar populations give traces of the formation history of the bulges. The metallicity distribution of K-giants in the Galactic bulge resembles to that of the giant ellipticals. There seems to be no conspicuous colour-magnitude relation intrinsic to the bulges. This can be explained if the bulges formed by the dissipative collapse of central regions of proto-galaxies followed by the supernova-driven bulge wind which was induced later than the dwarf ellipticals of the similar mass (the biased wind). Unfortunately, the observational data available at present of stellar populations of the bulges are not yet sufficient to get a firm conclusion on the origin of the bulges.
\end{abstract}

Key words: population synthesis - colour-magnitude relation - metallicity distribution - bulge formation - disc galaxies

\section{Introduction}

To study star formation history of a galaxy, it is of vital importance to know what kind of stars are of it major constituents. Two different approaches have so far been applied to analyse the population structure of galaxies: One is called as an empirical population synthesis and the other is called as an evolutionary population synthesis. Both are referred to as the stellar population synthesis.

The empirical method is to synthesize spectra of galaxies by using stellar and/or cluster spectral libraries and tries to get the best fits to the observed spectra. The spectral library should include the stellar populations with different spectral types, luminosity classes, and chemical compositions (cf., Spinrad \& Taylor 1971; Faber 1972). If the cluster library is used, the synthesis can be performed by using the star clusters of various ages and metallicities (Bica \& Alloin 1986; Bica 1988). The best fit is usually seeked by a trial-and-error method (eg., Faber 1972) or by the sophisticated minimization method (eg., Schmidt et al. 1991). Quite often the solution with negative contribution of certain stellar groups results. To avoid this happen, some of astrophysical constraints have been employed. These conditions include 1) non-negative contribution, 2) smooth distribution along the main sequence on the HR diagram, and 3) simple metal enrichement history (eg., Pickles 1985; Bica 1988). The resulting best fitted solution could reveal the stellar population structure of galaxies at the present epoch and would make it possible to trace back the star formation history in galaxies, unless galaxies contain a 
significant amount of possible intermediate-age stars, in which case the empirical method suffers a serious problem of non-uniqueness (cf.; Schmidt et al. 1991). Unfortunately under the scheme of empirical method, it is impossible to predict the time variation of galaxy spectra as a function of lookback time. Major achievments have been accomplished by Spinrad \& Taylor (1971), Faber (1972), O'Connell (1976), Pickles (1985), Rose (1985), and Bica (1988).

The evolutionary population synthesis assumes a certain scenario of galaxy formation and evolution, and predicts chemical and photometric (spectroscopic) properties of galaxies under the context of the presumed star formation history (eg., Tinsley 1972; Bruzual 1983; Arimoto \& Yoshii 1986). The resulting theoretical predictions are then confronted with observational properties, and the validity of the adopted formation scenario is studied in detail. The fundamental parameters that describe particular star formation history include star formation rate (SFR), initial stellar mass function (IMF), and accretion rate (ACR). The best set of these parameters is seeked until a reasonable agreement is attained between the predictions and the observed properties.

The evolutionary method is further split into somewhat different two approaches. One is chemcial evolution study that predicts chemical compositions of gas and stars in galaxies by using theoretical stellar nucleosynthesis theory (eg., Schmidt 1959; Talbot \& Arnett 1971; Tinsley 1974; Hartwick 1980; Lacey \& Fall 1985; Matteucci \& François 1989). The other is photospectroscopic evolution study that synthesizes spectra of galaxies by using stellar evolutionary tracks and stellar (cluster) spectral-photometric libraries (Tinsley 1972; Bruzual 1983). Both approaches assume the same context of galaxy formation and evolution. By combining two approaches, it is possible to get the most plausible scenario of galaxy formation and history of star formation that explains the observed chemical and photometric properties entirely consistently (Arimoto \& Yoshii 1986). The method predicts the time variation of galaxy spectra as a function of lookback time. This makes it possible to confront the predictions with the observational data of the colours, the redshift distribution, and the faint galaxy counts of galaxies at cosmological distance, as well as the back ground radiation in the universe. Mile stones and recent extensive studies include Tinsley (1972), Bruzual (1983), Arimoto \& Yoshii (1986), Guiderdoni \& Rocca-Volmerange (1987), and Buzzoni (1989).

In this review article, we present a brief description of the results of the stellar population synthesis applied to galactic bulges.

\section{Origin of bulges}

The origin of bulges is controversial. They either could form by dissipative collapse of proto-cloud with low angular momentum or could form at the center of disc galaxies which captured smaller satelites like dwarf irregular galaxies. 


\subsection{Dissipative Collapse}

Eggen et al. (1962), based on the kinematic and metallicity correlations, suggested that the formation of the Galaxy occurred by collapse from a larger volume, with progressive metal enrichment as the collapse proceeded, leading to the formation of central bulge with somewhat less dissipatively and to the subsequent formation of disc by cloud-cloud dissipative collisions. The timescale of tre collapse has been a matter of debate. Eggen et al. (1962) concluded that the collapse must have taken place within a few free-fall timescales, while Yoshii \& Saio (1979) derived the much longer timescale, of order $1 \mathrm{Gyr}$, from the same observational data used by Eggen et al. (1962). The 2D-hydrodynamical collapse model by Hensler et al. (1992) shows that already after a few free-fall timescales a clearly distinct central spheroid forms. A progressive shrinkage, spinning up, and enrichment of a gas cloud would end up in forming the rapidly rotating metal-rich bulge.

\subsection{MERGERS}

Schweizer \& Seitzer (1988) have presented evidence that ripples occur not only in ellipticals but also in disc galaxies of type S0, S0/a, and Sa, and probablly even in Sbc galaxies. The geometry of ripples suggests that the matter consisting of ripples is likely to be external in its origin. Since the lifetime of ripples is rather short, of order $1-2 \mathrm{Gyr}$, the capture or the merger event producing these ripples must happen recently. The numerical simulations indicate that the resulting merger remnants would look like ellipticals if merging disc galaxies are of nearly equal mass (Toomre \& Toomre 1972). This suggests that the ripples in disc galaxies must have been produced by a capture of a small galaxy by a massive disc galaxy. Stellar discs seems to be less fragile than is often thought. Since the stars of infalling galaxy will be trapped not by the disc of the main galaxy but by its bulge and halo, the presence of ripples in disc galaxies suggest that their bulges must have experienced episodic mass growth (Schweizer \& Seitzer 1988). Therefore, it is possible that bulges of some disc galaxies may be accumulated from the merger of smaller systems such as globular clusters or dwarf irregular galaxies. If the bulge mass grows gradually with a timescale comparable to the Hubble time (slow merging), a bulk of bulge stars must be composed of intermediate-age stars. On the other hand, if most of accumulation occurred very early (rapid merging), it would be difficult to distinguish the subsequent evolution from the dissipative collapse models (Rich 1990).

\section{Ellipticals vs bulges}

Are bulges small ellipticals? In many respects bulges are very similar to ellipticals. Table I shows a list of characteristics that have been used in arguing on the origin of ellipticals. Some characteristics are common in both ellipticals and bulges (E\&B), while others are known either in ellipticals (E) alone or only in bulges (B). Among the properties shown in Table I, those concerning the stellar populations generally 
suggest the dissipative collapse as the origin of ellipticals, while the kinematics and structures of ellipticals could be regarded as supporting evidence for merger hypothesis. In Table I, (E) does not necessarily mean that bulges do not share that property with ellipticals, instead it means that the characteristic is not yet confirmed observationally in bulges. Therefore, the table is not complete, and it is possible that many of characteristics listed here are indeed shared by both ellipticals and bulges, although some of them, like the cluster systems, may not relevant for studying the origin of bulges.

TABLE I

Similarities between ellipticals and bulges

\begin{tabular}{lll}
\hline & observations & similarities \\
\hline \multirow{3}{*}{ Stellar population } & Colour-magnitude relation & E \\
& Colour gradients & E \\
& Intermediate-age stars & E\&B \\
& UV flux upturn & E\&B \\
& Metallicity distribution & B \\
Global structures & Isophotal deviations & E\&B \\
& Scaling laws & E\&B \\
& Anisotoropy & E \\
Substructures & Triaxiality & E\&B \\
& Shells \& ripples & E\&B \\
& Counter rotating cores & E \\
Emissions & Dust & E \\
& X-ray emission & E\&B \\
Activity & Radio emission & E \\
Cluster systems & Star bursts & E \\
Interactions with & Globular cluster systems & E \\
environments & Morphology segregation & E \\
& Colour evolution & E \\
& Butcher-Oemler effect & E \\
& Heavy elements in ICM & E\&B \\
\hline
\end{tabular}

\section{Traces of bulge formation}

We confine ourselves to studying the stellar populations of bulges. In spite of many observational studies, it is not yet clear if the stellar populations of bulges are identical to those of ellipticals. Here we discuss several important features relevent to the population synthesis study. 


\subsection{Stellar metallicity distribution}

The metallicity of bulge stars is not uniform. Rich (1988) analysed spectra of 88 K-giants in Baade's window, most of them are members of the Galactic bulge, and showed that the strong-lined bulge giants are metal-rich with respect to the solar neighbourhood stars. The metallicity distribution shows the range $-1 \leq[\mathrm{Fe} / \mathrm{H}] \leq$ +1 . The mean stellar metallicity is about twice solar, and in contrast to the local solar neighbourhood a sufficient number of relatively low metal stars are detected, indicating that there is no G-dwarf problem in the Galactic bulge. Geisler \& Friel (1990) enlarge the number of K-giant to a few hundreds and confirm Rich's result.

However, Frogel et al. (1990) have conducted the infrared photometry of the Galactic bulge M-giants and have found that the infrared colour-magnitude diagrams of the bulge giants show rather small dispersion in $J-K$ colour, and the range in metallicities that would be deduced from $J-K$ colour is $\Delta[\mathrm{Fe} / \mathrm{H}] \simeq 0.5$, thus considerablly smaller than that of Rich (1988). The infrared CO index at $2.29 \mu \mathrm{m}$ also show significantly smaller scatter, suggesting smaller dispersion in metallicity (Frogel at al. 1990). This may be due to the unidentified blanketing agents or peculiarities in the atmosphere of the bulge stars (Frogel \& Whitford 1987). Alternatively, since Rich's spectroscopic estimates for most metal-rich stars have to rely on the extrapolation of calibration scale, the spread in metallicity found by Rich (1988) may be overestimated.

The relative abundance ratio of the Galactic bulge stars has not yet been fully studied. Preliminary analysis by Barbuy (1992) has shown that bulge-like nearby local metal-rich stars have $[\mathrm{C} / \mathrm{Fe}] \simeq 0.0,[\mathrm{~N} / \mathrm{Fe}] \simeq 0.0$, and $[\mathrm{O} / \mathrm{Fe}] \simeq 0.0$ to 0.2 . Rather normal $[\mathrm{O} / \mathrm{Fe}]$ ratio seems to suggest that these stars were born from the gas which had already been contaminated effectively by the ejecta of type Ia supernovae (SNIa). It would be difficult to explain this if the bulge had formed before SNIa produced iron significantly, ie $\sim 1$ Gyr. However, it is not certian if these stars belong to the same bulge population as Rich's K-giants. Detailed analyses of oxygen abundance of bulge K-giants are required.

A confusing result is coming from the abundance distribution of the Galactic bulge planetary nebulae ( $\mathrm{PNe}$ ). PNe originating from stars with main sequence mass less than $2 M_{\odot}$ are classified as Type II, and as believed not to experience the second dredge-up during the AGB evolution. Their ages, on average, are thought to be older than 3 Gyr. Ratag et al. (1992) have analysed the abudance distribution of Type II PNe near the Galactic centre. The distribution of bulge PN oxygen abundance is very similar to that of non-bulge PNe in the local solar neighbourhood, the mean value of bulge PNe is only $20 \%$ higher, although the excess of objects in the higher abudance range is remakable. This apparently is in conflict to the metallicity distribution of K-giants. Other elements such as neon, sulfer, and argon also show the very similar distribution to that of non-bulge PNe. Only helium and nitrogen show systematically higher abundances than non-bulge PNe, but this is very likely due to the dredge-up and may not reflect the abundance 
distributions of progenitor stars.

How can we reconcile the $\mathrm{PNe}$ abundance distribution with that of K-giants measured by Rich (1988)? Do both objects reflect the real abundance distributions? Two points are to be emphasized. 1) The spreads in abundance distribution larger than the observational errors are observed both in $\mathrm{PNe}$ and $\mathrm{K}$-giants; however, the dispersion of $\mathrm{PNe}$ is significantly smaller than the bulge giants. 2) The oxygen distribution of disc PNe has a considerablly lower mean than the solar value. The mean of $\mathrm{PNe}$ is $50 \%$ lower, while the bulge $\mathrm{PNe}$ is about $40 \%$ lower, implying unidentified systematic calibration errors in PN abundance measurement. Possible causes are discussed by Ratag et al. (1992). Iternatively, it is possible that K-giants reflect the metallicity distribution of the nuclear bulge, while PNe indicate that of outer bulge, thus the difference between two distributions may come from the possible metallicity gradient in the Galactic bulge. Until we obtain more data of the metallicity gradients, it would be rather dangerous to use both $\mathrm{PNe}$ and K-giants metallicity distributions simultaneously as the constraints to chemical evolution study of the Galactic bulge.

\subsection{Colour-magnitude (Metallicity-Luminosity) Relation}

It is highly uncertain if bulges of disc galaxies show the identical colour-magnitude (CM) relation to that of elliptical galaxies. The problem comes from the fact that it is rather difficult to estimate how much the measured bulge light is contaminated by disc light.

Turnrose (1976) performed the population synthesis of nuclear regions of Sc galaxies based on the narrow-band spectrophotometry covering wavelength range $3300-10400 \AA$. With the stellar birth function of the form $B(m, t)=c m^{-x} e^{-t / \tau}$, he found that the Salpeter-like IMF and the SFR gradually decreasing in time with a characteristic timescale $\tau=3-6$ Gyr can reproduce the observed spectral energy distribution. No clear indication of the bulge metallicity - luminosity relation was given.

Pritchet (1977) analysed the spectra of nucler bulges of 7 galaxies, M31 (Sb), M32 (E2), M51 (Sc), M81 (Sb), M86 (E3), M87 (E0p), M94 (Sb), NGC3115 (E7/S0), and NGC5195 (Irr), of the wavelength range $4200-6800 \AA$ with a resolution $\Delta \lambda=15-50 \AA$. His population model suggested that the nuclear bulges of intrinsically luminous galaxies have quite similar late-type stellar populations. However, the metallicity - luminosity relation of bulges was not explicitly discussed.

It is well known that ellipticals and S0's share the idenitcal CM relation (Visvanathan \& Sandage 1977; Persson et al. 1979; Bower et al. 1992). Bower et al. (1992) have shown that the scatter of early-type galaxies about the CM relation is typically as small as $0.05 \mathrm{mag}$, of which $0.03 \mathrm{mag}$ can be accounted for by observational measuring errors alone, suggesting no intrinsic scatter. S0 galaxies show slightly larger dispersion than ellipticals. It is not clear whether the CM relation of S0 galaxies is due to (1) an intrinsic CM relation of their bulges or (2) differ- 
ent degree of disc light contribution to the galaxy light within the aperture size adopted in the observations.

Some evidence suggest that $M g_{2}$ indices of luminous S0 discs are greater than those of fainter ones (R.Bender, private communication). Since $J-K$ colours of bulges and discs of S0 galaxies are nearly identical, bulges and discs of S0's of identical luminosities are likely to have the similar metallicity (Bothun \& Gregg 1990 ), provided that $J-K$ is a good metallicity indicator for gas-poor galaxies (Arimoto \& Yoshii 1987). This suggests that bulges of more luminous S0's have higher metallicities, and thus redder colours, than those of less luminous ones.

It is Bica (1988) who first studied systematically the metallicity - luminosity relation of bulges of spiral galaxies. Based on the cluster spectra library of Bica \& Alloin (1986), Bica analysed spectra of nuclei of 169 galaxies, of which 85 galaxies are spirals, in the wavelength range $3700-10000 \AA$ at $12 \AA$ resolution. According to characteristic features in spectra, spiral galaxies were binned into seven different groups S1 - S7. The groups S1 - S4 migrate simultaneously towards lower luminosity and later morphological type. These are reddest spiral groups in his sample and their spectra are very similar to the red elliptical groups E1-E4 (ie, without any significant evidence for star forming activity). The best fitted solutions were obtained by assuming simply that the metallicity of galaxy nucleus increases monotonically as a function of time. The solutions indicate that the maximum metallicities reach values a factor 4 solar (S1), 2 solar (S2, S3), and solar (S4). Bica thus suggested that the maximum metallicity of stars in the nucleus is related to the bulge luminosity.

If the bulge's metallicity - luminosity relation exists, as Bica suggested, it is interesting to see whether the relation is identical to that of ellipticals. In Fig.1 we plot a sum of equivalent widths of $\mathrm{CN}$ lines at $4200 \AA$ and of $\mathrm{Mg}+\mathrm{MgH}$ lines at $5175 \AA, W(C N, M g+M g H)$, as a function of total absolute magnitude by using Bica's S1-S4 spirals. Approximate locations of ellipticals, E1-E4, are also indicated for comparison. Surface decomposition parameters are not available for most of spirals shown in Fig.1; thus we are obliged to plot the total absolute magnitudes instead of bulge's intrinsic ones. Therefore, spirals should locate left to ellipticals even if the bulge's metallicity - luminosity relation is identical to that of ellipticals. In Fig.1, the scale given at the lower-left corner indicates the amount of luminosity decrease as a function of $L_{B} / L_{D}$ - the bulge-to-disc light ratio. $L_{B} / L_{D}$ amounting to 0.1 at $W(C N, M g+M g H) \sim 10 \AA$ and to 0.5 at $W(C N, M g+M g H) \sim 20 \AA$ could explain the apparent shift of S1-S4 spirals with respect to E1-E4 ellipticals. Although the precise comparison is impossible due to a lack of bulge's intrinsic luminosity, one can point out the followings: 1) there are very few spirals showing the line strengths as strong as giant ellipticals of metal-rich E1 group, 2) few spirals show such weak equivalent widths as dwarf ellipticals belonging to E4 group. If the spread in these line widths is due to the metallicity of bulge stars, the bulge metallicity is restricted to the range much smaller than that of ellipticals. This means that the bulge's metallicity - luminosity relation, if it exits, is not exactly 


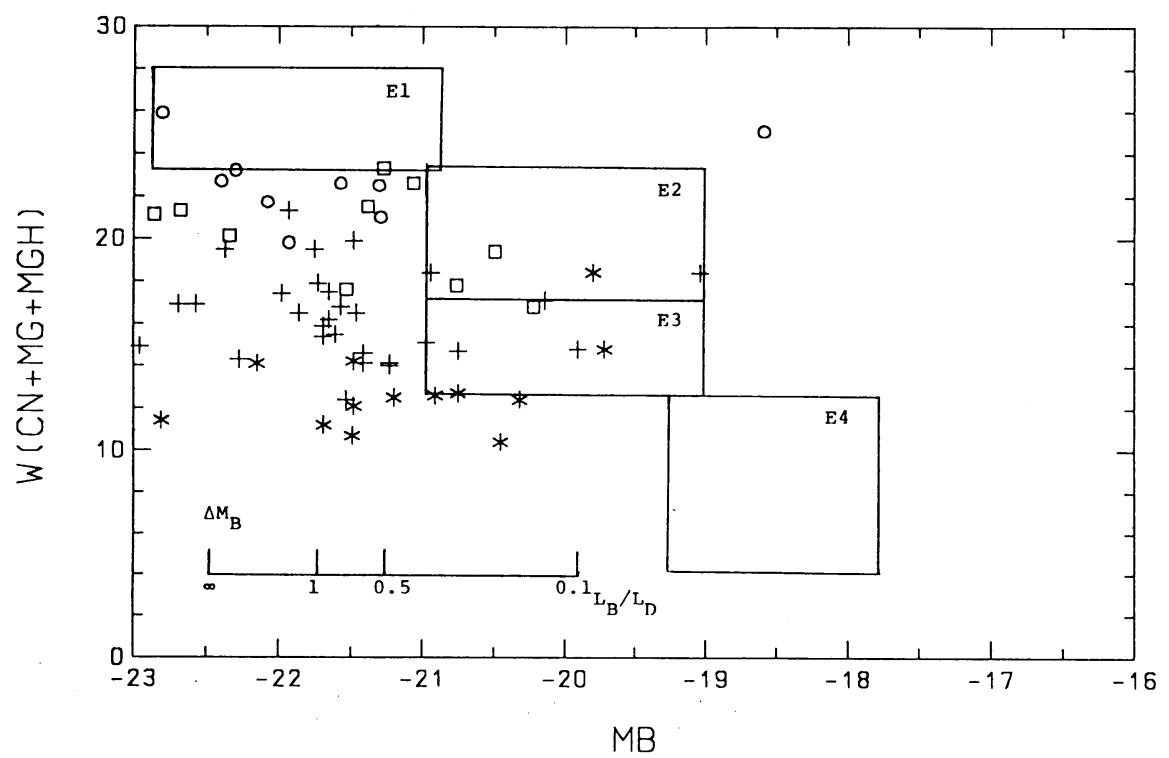

Fig. 1. Equivalent widths ( $\AA$ units) of $\mathrm{CN}, \mathrm{Mg}$, and $\mathrm{MgH}$ lines verse total absolute blue magnitudes for Bica's (1988) red spirals: S1 (open circles), S2 (open squares), S3 (crosses), S4 (asterisks). Four boxes indicate the regions where Bica's red ellipticals E1-E4 locate. The scale shown in the lower-left corner gives the amount of luminosity decrease for various values of the bulge-to-disc light ratio.

the same as that of ellipticals.

Although the aperture size used by Bica (1988) is relatively small, $0.8-1.4$ $\mathrm{Kpc}$ in average, it is not clear if there is no contamination of light from disc stars. His solutions show rather strange metallicity distribution of old stars (Table 4 in Bica). The solutions for S1-S4 all show the sharp peak and sudden drop at the higher metallicity end. This does not change when the luminosity contribution is converted into the mass contribution (Bica, Arimoto, Alloin 1988). Bica's metallicity distribution is completely different from that of bulge giants measured by Rich (1988), and such metallicity distribution is rather unexpected from the view point of chemical evolution. An exception is a galactic wind model, which predicts a sharp peak at the higher metallicity end (Yoshii \& Arimoto, 1987), if the wind expells gas before the maximum stellar metallicity exceeds the yield of nucleosynthesis. However, it is more likely that Bica's metallicity distribution of old stars is an artifact, mainly due to the very simplified astrophysical constraint adopted for the metal enhancement history. The monotonous metallicity increase is assumed in the bulge, but since the observed spectra may be contaminated by disc light, at least two different enhancement histories must be incorpolated in seeking the 
solution (cf., Arimoto \& Jablonka 1992).

It is essential to decompose the bulge and disc components of Bica's (1988) S1-S4 spirals and to evaluate the absolute magnitudes of bulges. This will make it possible to argue precisely the bulge metallicity - luminosity relation. Once such relation, if any, is established, we would be able to discuss conclusively whether bulges are small ellipticals of the same origin.

\section{Theoretical considerations}

\subsection{METAllicity Distribution}

The metallicity distribution of stars is sensitive to three fundamental parameters of star formation history; ie., the SFR, the IMF, and the ACR (Köppen \& Arimoto 1990). If the accretion is much faster than the star formation, the distribution is rather broad and peaks at $Z_{F e}=y_{F e}$, as can be shown easily for the simple closed-box model too. If the ACR is the same order as the SFR, the number of low metallicity stars is diminished, which is why infall models solve the G-dwarf probelm, but still show a peak very close to $Z_{F e}=y_{F e}$. However, if the ACR is slow enough, the distribution becomes markedly different: It rises towards higher metallicity monotonously, but is cut at $Z_{F e}=y_{F e}$, where it also has its maximum value (see Fig.2a of Köppen \& Arimoto 1990). Therefore, from the metallicity distribution of stars in the Galactic bulge, one can learn directly the yield of stellar nucleosynthesis there. This yield shows the efficiency of iron production by SNII if the timescale of bulge formation is shorter than $1 \mathrm{Gyr}$, and shows the combined efficiency by both SNII and SNIa if the bulge formation lasted longer than the effective lifetime of SNIa, ie., 1 Gyr (cf., Matteucci \& Brocato 1990).

Unfortunately, the stellar metallicity distribution alone cannot provide a crucial criterion distinguishing two controversial hypotheses. The metallicity distribution of the dissipative collapse model is essentially the same as that of the so-called simple model (closed one-zone, time and space constant IMF, initially metal-free). Tinsley \& Larson (1979) have shown that the metallicity distribution of merger models becomes identical to that of the simple model if two pre-merging subunit galaxies are identical. The metallicity distribution does not differ too much from the simple model even if Tinsley \& Larson's condition is relaxed (Arimoto \& Jablonla 1993, in preparation).

\subsection{RELATION}

So far there has been no theoretical consideration on the CM relation of the bulges. This is mainly because the bulge's CM relation has not been clearly identified. Since the CM relation of elliptcials has played a fundamental role in arguing their origin, we briefly describe theoretical models for the CM relation of ellipticals and introduce a theoretical attempt to explain the $\mathrm{CM}$ relation of the bulges. 


\subsubsection{Galactic wind models}

The CM relation of ellipticals can be understood in terms of increasing metallicity with luminosity (or equivalently mass). One possible explanation for this metallicity - mass relation is a galactic wind induced by supernova explosions during the dissipative collapse phase of galaxy formation (cf., Larson 1974; Arimoto \& Yoshii 1987; Matteucci \& Tornambè 1987). Following Carlberg's (1984) dissipative collapse model, Arimoto \& Yoshii (1987) constructed models which assume that an elliptical was initially a homogeneous gas sphere in which star formation was triggered by cloud-cloud collisions. A galactic wind is assumed to happen when the thermal energy provided by SNe exceeded the binding energy of the remaining gas. With a wind, the residual gas was swept away and the star formation was to stop. Because of the larger binding energy, the epoch of wind was delayed in an elliptical of larger initial mass. As a result, the mean stellar metallicity increased with the initial galaxy mass. The latest wind blew at $0.85 \mathrm{Gyr}$ in the model for giant elliptical galaxy of initial mass $210^{12} M_{\odot}$. Therefore, Arimoto \& Yoshii's wind models imply that almost all iron in ellipticals hàd been produced by SNII explosion. Needless to say, a bulk of subsequent iron formation should follow in ellipticals after the wind because of the delayed SNIa explosions (Matteucci \& Tornambé 1987). The lack of gas in ellipticals and the exsistence of hot X-ray halo around ellipticals and hot gas in clusters of galaxies suggest that iron from SNIa is now in the outside of ellipticlas.

\subsubsection{Merger models}

The other possibility to explain the metallicity - mass relation is the mergers (Tinsley \& Larson 1979; Arimoto \& Jablonka 1993). Elliptical galaxies are assumed to form by the hierarchical mergers of smaller gas-rich systems. It is assumed that stars to form only when star bursts are induced during violent mergings. If (only if) the SFR per unit mass is increasing in proportion to the total galaxy mass with the power-law index $p=1 / 3$, the observed metallicity - mass relation is reproduced. Tinsley \& Larson (1979) assumed the mergers of the identical gas-rich galaxies; later Arimoto \& Jablonka (1993) have studied chemical evolution of more generalized mergers by taking into account various combinations of pre-merger units whose star formation histories differ considerablly. The resulting $M g_{2}$ verse $M_{V}$ relation is shown in Fig.2. Although the merger process itself is quite stochastic, the resulting metallicity - mass relation shows surprisingly small dispersion at a fixed luminosity. Thus it is of no surprise that the CM relation of ellipticals does not show any significant dispersion (Bower et al. 1992).

However, there is one difficulty to be overcome in the merger hypothesis. The gas fraction in the merger remnant is too large to be an elliptcial (Fig.3). The chemical evolution of the merger is essentially given by the simple model (Tinsley \& Larson 1979). Therefore the merger remnant of small mass, having low metallicity, still keeps significant amount of residual gas. This amounts to $70 \%$ in the remnant of $10^{10} M_{\odot}$, and to $10-20 \%$ even in the most massive remnant of $10^{12} M_{\odot}$. If 


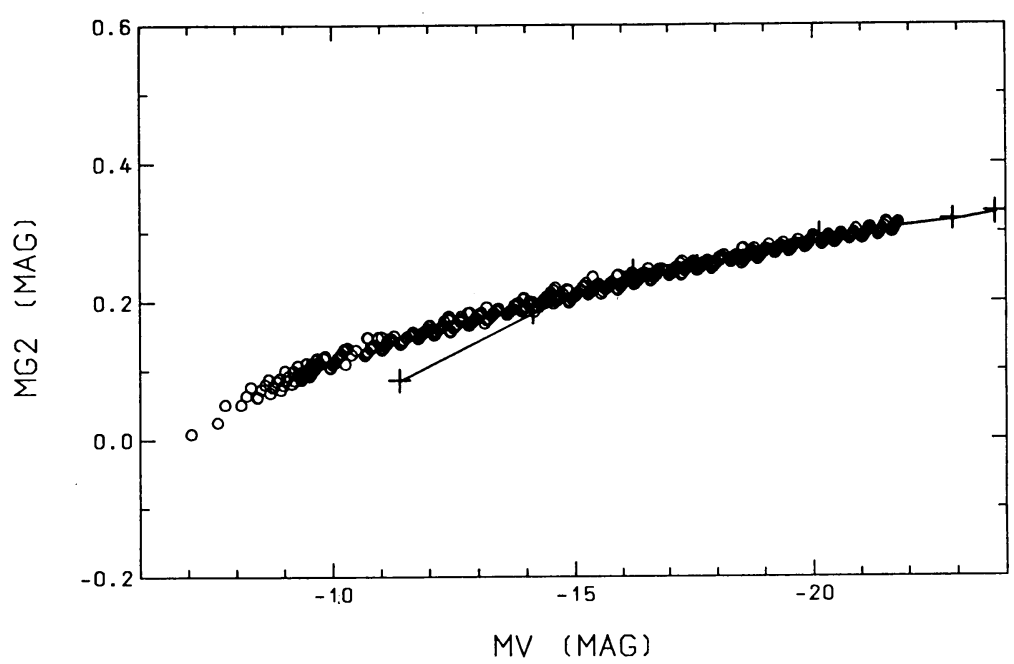

Fig. 2. The $M g_{2}$ verse $M_{v}$ relation of the stochastic merger models (open circles) by Arimoto \& Jablonka (1993). Crosses linked by a solid line indicate the galactic wind models by Arimoto \& Yoshii (1987) for comparison.

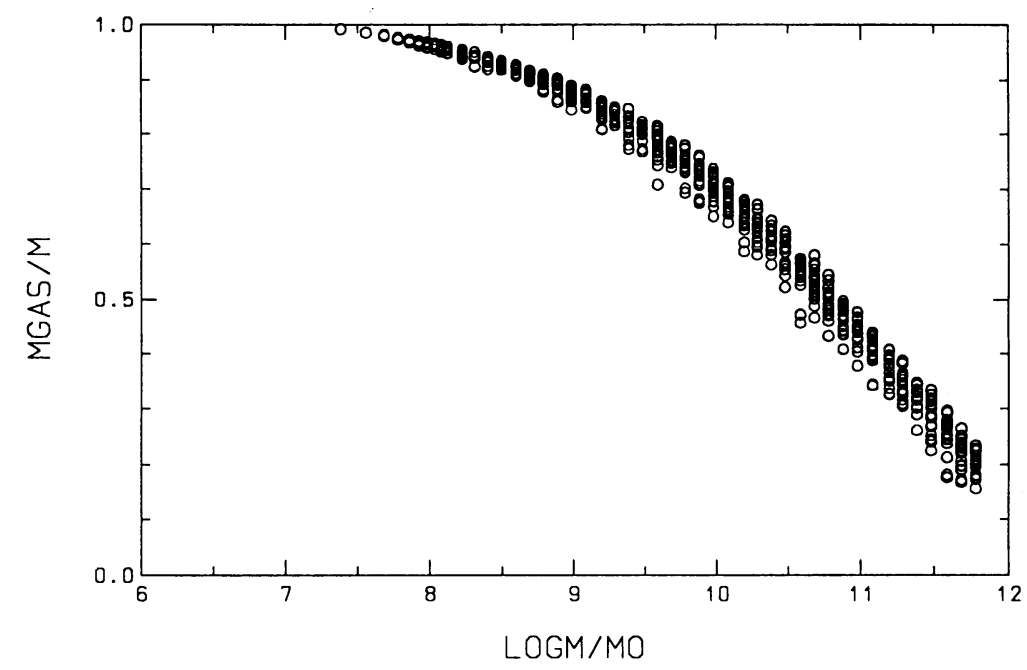

Fig. 3. The residual gas mass fraction of the merger models by Arimoto \& Jablonka (1993). The upper envelope corresponds to the twin mergers (mergers of identical galaxies) originally developed by Tinsley \& Larson (1979). 
the residual gas is further converted into stars gradually after the star bursts and eventually is used up completely in forming stars, the resulting stellar metallicity in the merger remnants should be indetical regardless of their mass. This would destroy completely the metallicity - mass relation. Therefore, the residual gas must be removed from the remnant. Since ellipticals do exist in both field and clusters, the gas removal mechanism must be intrinsic. The most plausible mechanism is an SN-driven wind. However, Arimoto \& Jablonka (1993) have found that the thermal energy release associated with a single star burst is not sufficient to induce a wind. The thermal energy at peak is at most one-tenth of the binding energy, and cools quickly once the star burst activity declines. Therefore, to induce a wind the successive events of mergers are required to happen within a short timescale before SN-ejecta of the previous generations cool down. Rough estimate suggests that the whole process must be completed within the same timescale of galaxy formation as that of Arimoto \& Yoshii's (1987) wind model, ie., 1 Gyr. In this case it would be rather difficult to distinguish between the wind models and the merger ones.

\subsubsection{Biased galactic wind}

Although not conclusive, there are several lines of evidence suggesting rather weak or no CM relation for the bulges of spirals and S0's: 1) Nuclei of spirals (S1S4; Bica 1988) show the narrow range in the equivalent width of metallic lines, $W(C N, M g+M g H) \simeq 10-24$, while normal red ellipticals (E1-E4) show much wider spread, $W(C N, M g+M g H) \simeq 5-30$ (Fig.1). This implies that the bulges of spirals have more or less similar metallicity regardless of their mass. 2) The optical - infrared colours of the brightest S0's are identical to those of giant ellipticals (Persson et al. 1979). Since discs would hardly contribute to the lights of these luminous S0's, this indicates that the bulges of luminous S0's show the same colours as giant ellipticals, in spite of the fact that masses of S0's bulges are smaller than giant elliptcials. 3) The metallicity distribution of K-giants in our Galactic bulge (Rich 1988) is very similar to that of the galactic wind model of a giant elliptical with the initial mass $10^{12} M_{\odot}$ (Arimoto \& Yoshii 1987). This metallicity distribution can be reproduced only if chemical evolution within the bulge is nearly complete (ie., at least more than $90 \%$ of gas must be converted into stars), although the mass of the Galactic bulge is far much smaller than giant ellipticals. The facts (1) - (3) seem to suggest that the stellar metallicities of the bulges of spirals and S0's are, irrespective of their mass, nearly identical to those of metal-rich giant ellipticals.

The mass of bulges is in the similar range to that of dwarf ellipticals. Nevertheless, metallicities of bulge stars are much higher than stars in dwarf ellipticals. The mean stellar metallicity of K-giants in our Galactic bulge is at least as high as twice solar, while that of dwarf ellipticals is at most solar or much less (cf., Yoshii \& Arimoto 1987). This could be explained by the biased bulge wind. At an epoch of SN-driven wind, the bulge must have been surrounded by the halo in which the 
primordial gas had been still abundant. In such a case the condition for the wind must be written as:

$$
E_{t h}=E_{b}+\int P_{e x} d V
$$

where $E_{t h}$ is the thermal energy of gas heated up by SN explosions, $E_{b}$ is the binding energy of gas within the bulge, and $P_{e x}$ is the external pressure of halo gas around the bulge. The second term $\int P_{e x} d V$ gives the amount of work that the bulge gas is required to do. This term is virtually negligible for ellipticals. Therefore, to induce a wind, the bulge must provide more thermal energy compared to ellipticals of the same mass. A larger number of SNe must have been exploded before the delayed wind was eventually triggered. The amount of gas that was converted into stars must be larger in the bulge than in the dwarf elliptical. This could explain why the bulges have higher metallicities, redder colours, and brighter surface brightness (because they lose smaller amount of gas) compared with the dwarf ellipticals (cf., Burkert \& Arimoto 1993, in preparation).

\section{Bulge model}

Under the context of dissipative collpase hypothesis, Arimoto \& Jablonka (1991) have constructed a bulge model that reproduces the metallicity distribution of K-giants obtained by Rich (1988). Within an uncertainty of measurements, the overall shape of the metallicity distribution of the Galactic bulge stars is best reproduced by the infall model that is characterized by a slope of the IMF $x_{B}=$ 1.05 (corresponding to the yield of three times solar), the SFR per unit mass $k_{B}=10 \mathrm{Gyr}^{-1}$, and the ACR $a_{B}=8 \mathrm{Gyr}^{-1}$. A bulge wind is assumed to occur at $t=0.7 \mathrm{Gyr}$, according to Arimoto \& Yoshii's wind model for a giant elliptical of an initial mass $10^{12} M_{\odot}$.

TABLE II

Bulge model properties

\begin{tabular}{llllllllll}
\hline$f_{g}$ & {$[\mathrm{Fe} / \mathrm{H}]_{\ell}$} & $U-B$ & $B-V$ & $V-R$ & $V-I$ & $V-J$ & $V-H$ & $V-K$ & $V-L$ \\
\hline 0.054 & +0.210 & 0.682 & 1.039 & 0.939 & 1.788 & 2.330 & 3.042 & 3.259 & 3.356 \\
\hline
\end{tabular}

Table II gives the resulting properties of the bulge model. In the $U-B$ verse $B-$ $V$ diagram, the bulge model locates at the reddest (lower-right) end of a sequence defined by normal galaxies, showing almost identical colours to giant ellipticals. By integrating over the observed luminosity function of stars in the Galactic bulge, Terndrup et al. (1990) have found that M-giants contribute significantly to the total light of the Galactic bulge and have obtained the infrared colours of the galactic bulge as $V-K=3.32, J-K=0.86$, and $H-K=0.21$, in excellent 
agreement with theoretical values $V-K=3.26, J-K=0.93$, and $H-K=0.22$ given in Table II. Bothun \& Gregg (1990) have measured optical-infrared colours of the bulges of S0's. After correcting the contamination of disc lights, they obtain $B-H=4.05 \pm 0.25$ and $J-K=0.92 \pm 0.08$ as mean values of their sample galaxies. Table II gives $B-H=4.08$, thus showing nearly identical colours to Bothun \& Gregg's value.

We note that once the bulge model is constructed by using chemical properties of the bulge as constraints, its photometric properties show an excellent agreement with the observed ones. As a conclusion, we stress that the dissipative collapse hypothesis is quite successful in reproduing both chemical and photometric properties of the bulges of spirals and S0's.

\section{Application to spiral galaxies}

A lack of a clear indication of the bulge's metallicity - luminosity relation suggests that the star formation history has been nearly identical in bulges of all spirals (and probablly of S0's) regardless of their luminosities and morphological types. The available data of bulge colours, as we have seen in section 6 , seem to support this unique bulge hypothesis. By using the bulge model discussed above, Arimoto \& Jablonka (1991) have built two component - bulge and disc - models of spirals to check if the unique bulge hypothesis brings any serious discrepancies between the theoretical predictions and the observed properties of spirals to which more various data are availabe than to the bulges. It is assumed that central regions of a proto-galaxy had collapsed rapidly and had formed the bulge, followed by the infall of loosely bound materials in the halo onto the equatorial plane and formed the disc. The star formation in the bulge stopped when the bulge wind ejected the residual gas containing the processed heavy elements into the halo. The ejecta were mixed with the primordial gas in the halo; therefore the gas infalling later onto the disc was pre-enriched.

TABLE III

Disc model properties

\begin{tabular}{lccccccccc}
\hline Type & {$[\mathrm{Fe} / \mathrm{H}]_{\ell}$} & $U-B$ & $B-V$ & $V-R$ & $V-I$ & $V-J$ & $V-H$ & $V-K$ & $V-L$ \\
\hline & & & & & & & & & \\
$\mathrm{Sa}$ & 0.061 & 0.037 & 0.665 & 0.770 & 1.538 & 2.054 & 2.784 & 2.961 & 3.061 \\
$\mathrm{Sb}$ & -0.095 & -0.055 & 0.575 & 0.691 & 1.353 & 1.817 & 2.464 & 2.659 & 2.761 \\
$\mathrm{Sc}$ & -0.209 & -0.125 & 0.505 & 0.629 & 1.207 & 1.619 & 2.196 & 2.393 & 2.496 \\
$\mathrm{Sd}$ & -0.333 & -0.154 & 0.474 & 0.607 & 1.163 & 1.566 & 2.116 & 2.328 & 2.430 \\
\hline
\end{tabular}

In a similar way to the bulge model, the parameters are fixed for each morphological type by using the observed characteristics of chemical properties, such as the stellar metallicity distribution and the hydrogen mass to stellar luminosity ra- 
tio, of the discs of spiral galaxies. The following values are chosen: the IMF slope $x_{D}=1.45$ (the yield corresponds to a half solar), the ACR $a_{D}=0.17 \mathrm{Gyr}^{-1}$, and the SFR $k_{D}=0.69,0.32,0.25,0.17 \mathrm{Gyr}^{-1}$ for the type $\mathrm{Sa}, \mathrm{Sb}, \mathrm{Sc}$, and $\mathrm{Sd}$, respectively. The resulting properties of the disc models are given in Table III.

Assuming de Vaucouleurs' $r^{1 / 4}$-law for the surface brightness distribution of the bulge and an exponential-law for the disc, the local bulge-to-disc light ratios are calculated in B-band as a function of the aperture size for 79 spirals to which decomposition parameters of the surface brightness profile are empirically known (Simien \& de Vaucouleurs, 1986; Kent, 1985). The local bulge-to-disc ratios are then used as a weight for synthsizing the photometric properties of individual 79 spirals. Regarding these spirals as a sample of normal spirals of different morphological types, we have compared the model properties with the observed ones. It has been shown that the disc mass of spirals is confined in rather a small range around $10^{11} M_{\odot}$ (a Hubble constant $H_{0}=50 \mathrm{~km} \mathrm{~s}^{-1} \mathrm{Mpc}^{-1}$ is assumed), while the bulge mass shows clear decrease from $\sim 310^{11} M_{\odot}$ to $\sim 310^{9} M_{\odot}$ towards later types along the morphological sequence, although the dispersion at a fixed type amounts to one order of magnitude.

The bulge-to-disc ratio is the single dominant parameter that leads to narrow distributions of spirals on the colour-colour diagrams (such as $U-B$ verse $B-V$ ) and that leads to general trends of optical-infrared colours along the morphological sequence. The scatter in colours of spiral at a fixed type arises from the intrinsic scatter in the bulge-to-disc ratio. This clearly demonstrates that if the bulgeto-disc ratio is chosen as a major factor of galaxy classification the photometric properties of spirals would correspond uniquely to the classified type. As is clearly shown in Tables II and III, the bulges and the discs have almost the same $J H K$ colours; as a result, the near-infrared colours do not depend on the bulge-to-disc ratio and therefore show no clear trend along the morphological sequence as was already pointed out observationally by Gavazzi \& Trincheri (1989). In early type spirals, S0/a-Sab, the CM relation in the $U-V$ verse $M_{v}$ diagram has been known to exit (Griersmith 1980). This has been regarded as an evidence for the bulge's metallicity - luminosity relation. However, it is shown that this CM relation arises from a variation of the bulge-to-disc ratio and not from the bulge's metallicity - luminosity relation. Therefore we do not find any evidence that requires the metallicity - luminosity relation that is intrinsic to the bulges of spirals.

\section{Conclusions}

It is not clear whether the stellar populations of the bulges are identical to the ellipticals exists. Bica (1988) suggested an intrinsic CM relation of the bulges of normal spirals. However, it cannot be considered as real until one reanalyse his data by carefully removing the contaminated disc light and by introducing more realistic metal enhancement history in the bulges. Needless to say, accurate measurements of the bulge's intrinsic luminosities are required to be done by decomposing the 
surface brightness distribution into the bulge and disc components.

Apart from Bica's population synthesis study, there are no other works that suggest the $\mathrm{CM}$ relation of the bulges. Instead, the metallicity distribution of $\mathrm{K}$ giants in the Galactic bulge (Rich 1988) indicates that the star formation history must have been very similar to the giant ellipticals, although the mass of the former is much close to the dwarf ellipticals than to the latter. This suggests that chemical evolution in the bulges of any mass is nearly identical to the giant ellipticals. We therefore conclude that there is no conspicuous CM relation intrinsic to the bulges and that the stellar populations of the bulges are more or less identical to the giant ellipticals.

The bulges could form either by the dissipative collapse of the central regions of proto-galaxies or by the capture of smaller satelite galaxies by massive disc galaxies. Our present knowledge of the stellar populations of the bulges is confined to the stellar metallicity distribution and the $\mathrm{CM}$ relation, which is not sufficient to reveal the origin of the bulges. Observational data of the colour gradients would provide important constraint to the bulge formation. Search for the intermediate-age stars and a systematic study of the UV-flux from the bulges are also fundamental. The relative abundance ratios, such as $[\mathrm{O} / \mathrm{Fe}]$ and $[\mathrm{Mg} / \mathrm{Fe}]$, would eventually tell the timescale of star formation in the Galactic bulge. Of course more careful analyses of the stellar metallicity distribution as well as the CM relation would be fruitful.

\section{Acknowledgements}

I am grateful to P.Jablonka for her energetic contribution to our works on the population synthesis. R.Bender and R.Guzman kindly provided me their recent photometric data of elliptcials before publication. Special thanks go to F.Leeuwin for many discussions on the kinematics of the ellipticals and the bulges, without her contribution I could not make better approach to the problem. The University of Tokyo kindly allowed me to stay in Germany longer so that I could attend this fruitful meeting. Finally financial support from the Deutsche Forschunsgemeinschaft (SFB 328) is gratefully acknowledged.

\section{References}

Arimoto, N., Jablonka,J.: 1991, $A \& A$ 249, 374

Arimoto, N., Yoshii, Y.: 1987, $A \& A 173,23$

Arimoto, N., Yoshii, Y.: 1986, $A \& A$ 164, 260

Barbuy,B.: 1992, IAU Symp. No.149, The Stellar Populations of Galaxies, B.Barbuy, A.Renzini

Kluwer, Dordrecht 143

Bica, E.: 1988, $A \& A$ 195, 76

Bica, E., Arimoto, N., Alloin, D.: 1988, $A \& A$ 202, 8

Bica, E., Alloin,D.: 1986, $A \mathscr{G} A$ 162, 21

Bothun, G.D., Gregg, M.D.: 1990, ApJ 350, 73

Bower, R.G., Lucey, J.R., Ellis, R.S.: 1992, MNRAS 254, 601

Bruzual,G.A.: 1983, ApJ 273, 105 
Buzzoni,A.: 1989, ApJS 71, 817

Eggen,O.J., Lynden-Bell,D., Sandage,A.: 1962, ApJ 136, 748

Faber,S.M.: 1972, $A \& A$ 20, 361

Frogel,J.A., Terndrup,D.M., Blanco,V.M., Whitford,A.E.: 1990, ApJ 353, 494

Frogel,J.A., Whitford,A.E.: 1987, ApJ 320, 199

Gavazzi,G., Trincheri,G.: 1989, ApJ 342, 718

Geisler,D., Friel,E.: 1990, Bulges of Galaxies, B.Jarvis, D.Trendrup ESO 77

Griersmith,D.: 1980, $A J$ 85, 1295

Guiderdoni,B., Rocca-Volmerange,B.: 1987, $A \& A$ 186, 1

Hartwick,F.D.A.: 1980, ApJ 236, 754

Hensler,G., Burkert,A., Truran,J.W., Dünhuber,H., Theis,C.: 1992, The Stellar Population of Galaxies, B.Barbuy, A.Renzini

Kluwer, Dordrecht 119

Kent,S.M.: 1985, ApJS 59, 115

Köppen,J., Arimoto,N.: 1990, A\&B 240, 22

Lacey,C.G., Fall,S.M.: 1985, ApJ 290, 154

Larson,R.B.: 1974, MNRAS 166, 585

Matteucci,F., Brocato,E.: 1990, ApJ 365, 539

Matteucci,F., François,P.: 1989, MNRAS 239, 885

Matteucci,F., Tornambé,A.: $1987, A \& A$ 185, 51

O'Connell,R.W.: 1976, ApJ 206, 370

Persson,S.E., Frogel,J.A., Aaronson,M.: 1979, ApJS 39, 61

Pickles, A.J.: 1985, ApJ 296, 340

Pritchet,C.: 1977, ApJS 35, 397

Ratag,M.A., Pottasch,S.R., Dennfeld,M., Menzies,J.W.: 1992, AछA 255, 255

Rich,R.M.: 1990, ApJ 362, 604

Rich,R.M.: 1988, $A J$ 95, 828

Rose,J.A.: 1985, AJ 90, 1927

Schmidt,A.A., Copetti,M.V.F., Alloin,D., Jablonka,P.: 1991, MNRAS 249, 766

Schmidt,M: 1959, ApJ 129, 243

Schweizer,F., Seitzer,P.: 1988, ApJ 328, 88

Simien,F., de Vaucouleurs,G.: 1986, ApJ 302, 564

Spinrad,H., Tayler,B.J.: 1971, 'ApJS 2, 445

Talbot,R.J., Arnett,W.D.: 1971, ApJ 170, 409

Tinsley,B.M., Larson,R.B.: 1979, MNRAS 186, 503

Tinsley,B.M.: 1974, ApJ 192, 629

Tinsley,B.M.: 1972, A\&A 20, 383

Terndrup,D.M., Frogel,J.A., Whitford,A.E.: 1990, ApJ 357, 453

Toomre,A., Toomre,J.: 1972, ApJ 178, 623

Turnrose,B.E.: 1976, ApJ 210, 33

Visvanathan,N., Sandage,A.: 1977, $A p J$ 216, 214

Yoshii,Y., Arimoto,N.: 1987, $A \& A$ 188, 13

Yoshii,Y., Saio,H.: 1979, PASJ 31, 339 


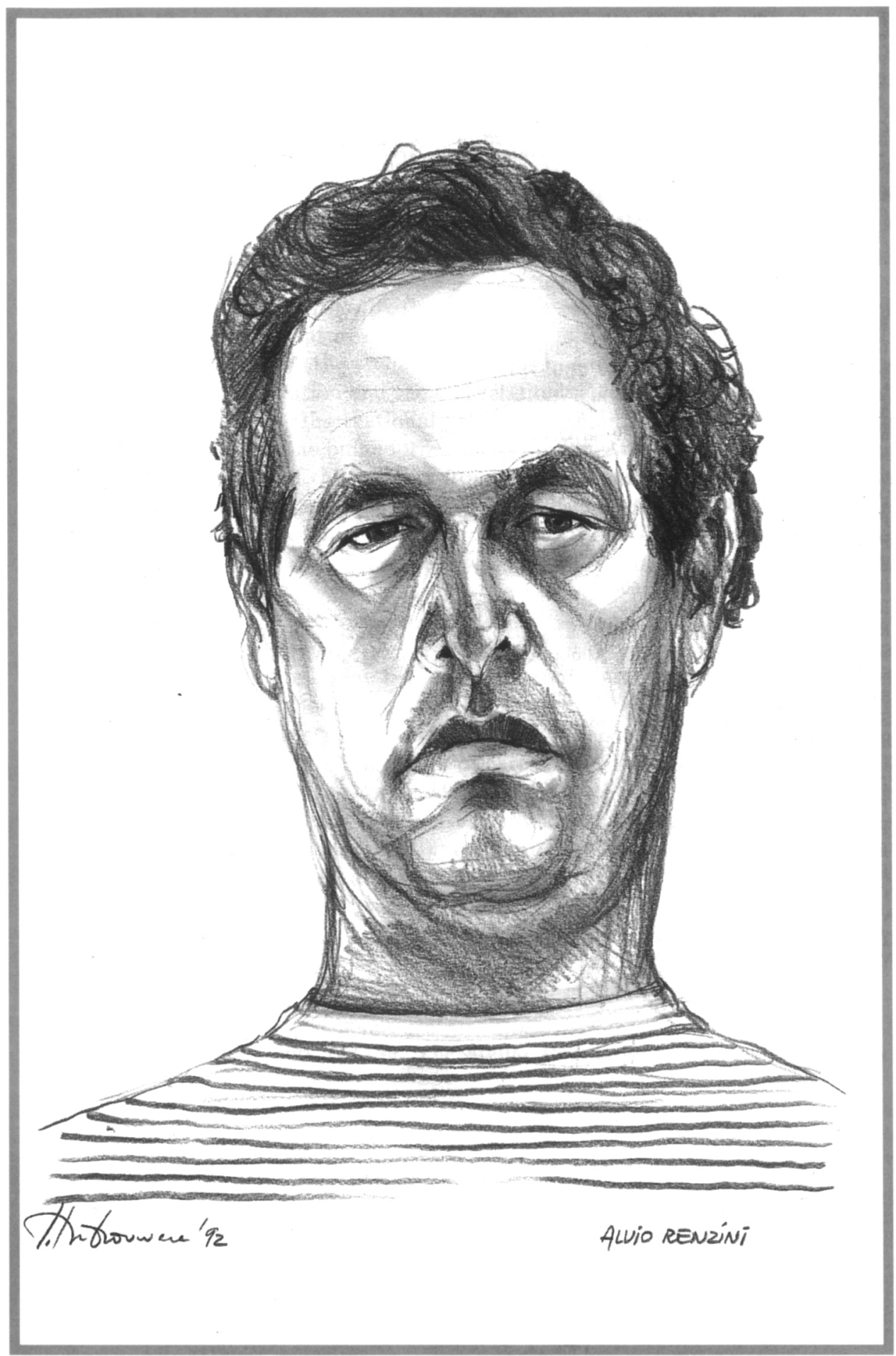

